\title{
$\mathrm{X}$.
}

\section{Ueber die giftige Wirkung der chlorsauren Salze.}

\author{
Zur Erwiderung an B. J. Stokvis in Amsterdam.
}

\author{
Von \\ F. Marchand \\ in Marburg.
}

Stokvis ${ }^{1}$ ) hat die giftige Wirkung der chlorsauren Salze von Neuem einer Untersuchung unterworfen und ist dabei zu Resultaten gelangt, welche von der bisher ziemlich allgemein angenommenen Ansicht ïber diesen Gegenstand in hohem Grade abweichen. Da ich bei der Begründung dieser letzteren nicht ganz unbetheiligt bin und dieselbe auch trotz der abweichenden Meinung Stokvis' im ganzen Umfang aufrecht erhalte, so sehe ich mich hier zu einer nochmaligen Aeusserung uber die Frage veranlasst.

Seit dem Jahre 1879 ist die Zahl der bis dahin ziemlich vereinzelt gebliebenen Publicationen uiber Vergiftungsfälle durch chlorsaures Kali ziemlich erheblich gewachsen. Es fehlte anfangs nicht an Meinungsverschiedenheiten uiber die Deutung des Befundes, die Ursache des Todes, es fehlte auch nicht an Mittheilungen praktischer Aerzte, welche zwar die giftige Wirkung des chlorsauren Kali in sehr grossen Dosen anerkannten, aber doch von der grossen Heilkraft des Mittels bei Diphtheritis und anderen Krankheiten so fest tiberzeugt waren, dass sie von Neuem stets das Kali chloricum als Panacee anpriesen, obne sich durch die notorische Gefährlichkeit des Mittels und die stets von Neuem vorkommende Vergiftungsfälle abschrecken zu lassen. ${ }^{2}$ )

Ich habe mich jenen Aeusserungen gegenüber nicht berufen gefühlt, das zu wiederholen, was ich vor 7 Jahren, wie mir schien,

1) Dieses Archiv. XXI. Bd. S. 169.

2) Erst vor Kurzem erhielt ich Kenntniss von einem neuen derartigen traurigen Fall, in welchem der Tod eines'Knaben die Folge ärztlicher Verordnung von Kali chlor. war. 
sicher nachgewiesen hatte. Ich habe auch jetzt nicht die Absicht, den therapeutischen Werth des chlorsauren Kali gegenüber seiner Giftigkeit abzuwägen, da ich weder Pharmakologe bin, noch auch eigene praktische Erfahrungen gesammelt habe. Ich kann indess beiläufig meine bescheidenen Zweifel an den angeblich glänzenden Erfolgen des Mittels bei innerem Gebrauch nicht ganz unterdrücken, da ich in diesem Punkte überhaupt etwas skeptisch bin. Dass die locale Anwendung des Salzes nicht zu unterschätzen und bei nöthiger Vorsicht auch ungefährlich ist, will ich gern anerkennen.

Näher betrafen mich - abgesehen von dem gewiss bedauerlichen Umstande, so und so viele Menschenleben durch Unvorsicht, Unkenntniss, um nichts Schlimmeres zu sagen, vernichtet, oder wenigstens ernstlich bedroht zu sehen - die Meinungsverschiedenheiten uiber die Ursache der giftigen Wirkung der chlorsauren Salze anf den Organismus. Ich erwähne hier u. A. die Annahme von Leichtenstern ${ }^{1}$ ), dass bei den ganz acut verlaufenen Vergiftungsfällen die Kaliwirkung die Hauptrolle spielt; diese Annahme ist indess hinfällig, da ich bereits gezeigt hatte, dass die Natriumverbindung sich ebenso giftig verhält wie das Kalisalz. Dass noch manche unaufgeklärte Widersprüche in dieser Frage bestanden, namentlich der oft behauptete Uebergang des eingeftihrten Salzes in den Urin in ganz unzersetztem Zustande, ferner die sehr wechselnde Giftwirkung je nach den Umständen und je nach der Thierart, der unschädliche Gebrauch grosser Dosen des Mittels in dem einen, und die schnell tödtende Wirkung verhältnissmässig kleiner Gaben in dem anderen Falle, habe ich mir schon damals nicht verhehlt, musste aber die zu erwartende Aufklärung über diese Punkte einer späteren Zeit überlassen.

Inzwischen hat $\nabla$. Mering ${ }^{2}$ ) diese Fragen, als Pharmakologe und Chemiker weit besser hierzu vorbereitet als ich, von Neuem in Angriff genommen und dieselben auch wesentlich gefördert.

Stokvis ist nun in seiner Arbeit zu Schlüssen gelangt, welche in der That in so diametralem Gegensatz zu dem stehen, was ich auf Grund von Thierversuchen und Sectionsbefunden als sichergestellt annehmen zu müssen glaubte, und was, meiner Meinung nach, durch die häufigen seitdem vorgekommenen Vergiftungsfälle beim Menschen auch vollkommen bestätigt worden ist, dass in der That

1) Deutsche medicin. Wochensehrift. 1884. Nr. 4.

2) Das chlorsaure Kali, seine physiologischen, toxischen und therapentischen Wirkungen. Berlin 1885 . 
wenig mehr davon übrig bleiben würde, als die einfache Thatsache, dass in dem ,todten" Blut durch Gegenwart von chlorsauren Salzen das O-Hämoglobin die Umwandlung in Methämoglobin erleidet. „Es ist mir hoffentlich der Beweis gelungen", sagt Stokvis am Schlusse seiner Arbeit S. 216, „dass von einer besonderen eigentlichen toxischen Wirkung der chlorsauren Alkalisalze keine Rede sein kann, und dass die Giftwirkung dieser Substanzen theils der irritirenden (resp. corrodirenden) Wirkung jeder stark concentrirten Salzlösung, theils den physiologischen Wirkungen ihrer alkalischen Componenten zugeschrieben werden muss".

St okvis geht von der Annahme aus, dass die chlorsauren Salze den Körper ganz oder fast ganz unzersetzt passiren, und dass dieser Umstand mit dem Zustandekommen der von mir behaupteten eigenthümlichen Blutveränderung im lebenden Körper - der Umwandlung von 0-Hämoglobin in Methämoglobin - unverträglich sei, da durch diese nothwendigerweise eine 0 -Abgabe vorausgesetzt werde. Die erstere Annahme sucht Stokvis durch Urinuntersuchungen beim Menschen und bei Kaninchen zu beweisen, auf Grund derer er zu dem Schluss gelangt, dass ,eine Reduction der chlorsauren Salze im Organismus sich in keiner Versuchsreihe nachweisen liess". Ande ${ }_{\perp} \mathrm{er}-$ seits kam er, besonders durch Versuche mit Zusatz von chlorsaurem Kali zu Blut ausserhalb des Körpers und mit Rücksicht auf seine negativen Resultate am lebenden Kaninchen zu dem ergänzenden Schluss, ,dass die Vorgänge der Blutzersetzung unter dem Linflusse chlorsaurer Salze ausserhalb des Organismus am besten als Fäulnissvorgänge aufgefasst werden, dass endlich drittens eine Methämoglobinbildung im lebenden Blute selbst bei der Einfuhr grösserer und sehr grosser Dosen der Chlorate nicht angenommen werden" und dass demnach ,die toxische Wirkung der chlorsauren Salze unmöglich von einer durch sie verursachten Zersetzung des circulirenden Blutes abhängig gemacht werden kann" (S. 202). Nachdem Stokvis dies festgestellt $\mathrm{zu}$ haben meint, betrachtet er die noch ïbrigbleibenden Vergiftungserscheinungen, welche sich doch nun einmal nicht wegleugnen lassen, und gelangt bezigglich des chlorsauren Natron zu dem Resultat, dass dasselbe sowohl bei intravenöser Einverleibung als bei innerlicher Darreichung ,sich nicht mehr und nicht weniger giftig ergiebt, als das gewöhnliche Kochsalz" (S. 209), und bezüglich des chlorsauren Kali, ,dass demselben keine andere selbständige Wirkung auf den Organismus zugeschrieben werden kann als die, welche auch allen anderen Salzen und insbesondere allen Kalisalzen als solchen zukommt"s. 
Die Resultate, welche ich dagegen aus den Thierversuchen über die Wirkung der chlorsauren Salze in Verbindung mit den Sectionsergebnissen von Vergiftungsfällen beim Menschen erhalten hatte, fasse ich folgendermaassen zusammen:

Die chlorsauren Salze - gleichviel ob Kali oder Natron - wirken giftig durch eine directe Einwirkung auf das Blut, welche durch die Umwandlung des $O$-Hämoglobin in Methämoglobin charalterisirt wird. Die Blutkörperchen werden dadurch unfähig, der Athmung zu dienen, und gehen selbst dabei zu Grunde. Ueberschreitet diese Veränderung einen gewissen Grad, so kann der Tod unmittelbar als Folge derselben eintreten, und die einzige Veränderung, welche dann an der Leiche gefunden wird, ist die eigenthümliche dunkelbraune Verfärbung des Blutes.

Ist die Blutveränderung nicht hinreichend, um den Tod sofort herbeizuführen, so dass einige Zeit bis zu demselben verstreicht, so werden die zerstörten rothen Blutkörperchen theilweise in der Milz angehäuft, theilweise durch die Nieren ausgeschieden. Man findet infolge dessen in diesem Stadium eine sehr starke Schwellung der Milz durch enorme Anhäufung der Zerfallsproducte der rothen Blutkörperchen, gleichzeitig mit der eigenthümlichen Verfürbung, und namentlich die Erscheinungen der exquisiten Hämoglobin- resp. Methämoglobinausscheidung in den Nieren und im Urin. Daneben kann die dunkolbraune Färbung des Blutes noch bestehen, oder sie kann, je nach der Dauer der Vergiflung, bereits verschwunden sein.

Auch dieser Zustand kann überlebt werden, wofern die Alteration des Blutes nicht sehr intensiv war. Es kann nach Abscheidung der zerfallenen und zersetzten Blutkörperchen vollständige Wiederherstellung stattfinden.

Indess kann der Tod auch noch secundär eintreten, nachdem die ganze Blutveränderung und der eigentliche Ausscheidungsprocess abgelaufen ist, und zwar offenbar infolge der intensiven Verstopfung der geraden Kanälchen der Nieren durch die stark veränderten Hümoglobinmassen. Der Tod erfolgt in diesen Fällen unter cerebralen Erscheinungen infolge der Suppression des Urins, also unter dem Bilde der Urämie, oder, was ich als selbstverständlich noch hinzuftugen kann, an Entkräftung.

Man wird zugeben, dass ein grösserer Widerspruch zwischen dieser von mir aufgestellten Darstellung der toxischen Wirkung der chlorsauren Salze und der von Stokvis gegebenen kaum denkbar ist. 
Ich erlaube mir hier zunächst eine kurze Uebersicht der wichtigsten von mir angestellten Versuche an Hunden, dem Verlaufe nach geordnet, zusammenzustellen, wobei ich auch die später von L e be deff ${ }^{1}$ ) und mir gemeinschaftlich vorgenommenen mit aufnehme, da Stokvis die letzteren gar nicht berickssichtigt hat, wie er uberhaupt auf die Ergebnisse der bisherigen Versuche an Hunden ausserordentlich wenig Werth zu legen scheint.

\section{Versuch 1.2)}

Hund von 7 Kilo. $10 \mathrm{~g}$ Natr. chlor. intraperitoneal (1,43 pro Kilo). Beginnende Blutveränderung an dem aus der Arterie fiessenden Blute 46 Minuten, Tod 111 Minuten nach der Injection. Blut dunkel-chocoladenbraun; keine Milzschwellung oder Nierenaffection.

\section{Versuch 2.31)}

Hund von 8,3 Kilo. $10 \mathrm{~g}$ Natr. chlor. intraperitoneal (1,2 g pro Kilo). Beginnende Blutveränderung 58 Minuten, Tod 67 Minuten nach der Injection. Blut und Organe ebenso wie vorher.

\section{Versuch 3.4)}

Hund von 6 Kilo. $12 \mathrm{~g}$ Natr. chlor. in 3 Dosen in Zeit von $31 / 2$ Stunden innerlich ( $2 \mathrm{~g}$ pro Kilo). Tod nach 3 Stunden. Blut (unmittelbar nach dem Tode constatirt) dunkelbraun. Starke Milzschwellung, kaum beginnende Hglb.-Ausscheidung ${ }^{5}$ ) in den Nieren.

\section{Versuch $4 .{ }^{6}$ )}

Hund von 5 Kilo. $4 \mathrm{~g}$ Natr. chlor. subcutan in 2 Dosen (nach vorhergegangenen, grösstentheils ausgebrochenen inneren Gaben), Tod nach 5 Stunden. Starke Blutveränderung unmittelbar nach dem Tode constatirt; sehr starke Milzschwellung. Geringe Hglb.-Ausscheidung in Nieren und Urin.

\section{Versuch 5.7)}

Hund von 17,8 Kilo. $50 \mathrm{~g}$ Natr. chlor. in 5 Dosen innerlich $(2,8 \mathrm{~g}$ pro Kilo). Tod am folgenden Tage. Blut dunkel-chocoladenbraun. Colossale Milzschwellung, höchster Grad von Hglb.-Ausscheidung in Nieren und Urin.

\section{Versuch 6.9)}

Hund von 9 Kilo. In 8 Tagen kleine allmählich steigende Dosen; Starke Polyurie. Am 9 Tage $16 \mathrm{~g}$ in 8 Dosen $(1,7 \mathrm{~g}$ pro Kilo). Tod

1) Virchow's Archiv. 91, Bd. 1883, S. 274.

2) Ebenda. 77. Bd. S. 472.

3) Ebenda. S. 473.

4) Ebenda. 91. Bd. S 278.

5) Der Einfachheit wegen wird die Hämoglobin- und Methämoglobinausscheidung als Hglb.-Ausscheidung bezeichnet.

6) Virchow's Archiv. 91. Bd. S, 277.

8) Ebenda. 91. Bd. S. 274.

7) Ebenda. 77. Bd. S. 469. 
am folgenden Tage. Blut dunkel bräunlich, nicht vollständig sepiafarbig. Sehr starke Hglb.-Ausscheidung in Nieren und Urin. Milzschwellung.

\section{Versuch 7.1)}

Hund von 7,5 Kilo. $15 \mathrm{~g}$ Natr. chlor. in 3 Dosen (2 $\mathrm{g}$ pro Kilo) vom Nachmittag bis zum anderen Morgen. Am Nachmittag desselben Tages getödtet. Blut dunkel braunroth. Milz colossal vergrössert, reicht iiber die Mittellinie nach rechts. In der Blase reichliche dunkelbraune Massen. In den Nieren keilförmige Streifen von Hglb.-Ausscheidung.

Ich führe hier noch einen Versuch von Brenner ${ }^{2}$ ) an als Beispiel einer geheilten Kali chlor.-Vergiftung beim Hunde nach erfolgter Hglb.-Ausscheidung, welche mir besonders deswegen wichtig ist, weil Brenner gleichzeitig und unabhängig von mir die giftige Wirkung des chlorsauren Kali auf eine Blutzersetzung zurickfuhrte.

\section{Versuch.}

Bulldogge mittlerer Grösse. $15 \mathrm{~g}$ Kali chlor. in Lösung auf einmal innerlich, am Morgen. Mittags keine Fresslust; Nachmittag weniger munter. Heller Urin. Abends: Hund kauert im Winkel, seit mehreren Stunden kein Urin. In der Nacht schwarzer Urin. Am anderen Tage fortwährend Harndrang, wenige Tropfen dunklen Harns. Allmählich wurde der Urin wieder heller, mit demselben wurden noch Klümpchen von brauner Farbe entleert. Nach 6-7 Tagen vollständige Heilung.

Diese Versuchsergebnisse scheinen mir doch unwiderleglich genug zu beweisen, dass die Blutveränderung in der That das Erste und Wichtigste ist; darauf folgt die Anhäufung der Zerfallsmassen in der Milz (auch im Knochenmark) und die Ausscheidung durch die Nieren. Erfolgte der Tod sehr kurze Zeit nach der Vergiftung (Versuch 1 und 2), so fand sich lediglich die dunkelbranne Farbe des Blutes und der Organe; erfolgte er einige Stunden nach der subcutanen oder innerlichen Einfuhr, so fand sich starke Blutveränderung, bereits starke Milzschwellung, aber geringe (Versuch 4) oder kaum beginnende und erst mikroskopisch zu constatirende (Versuch 3) Hglb.-Ausscheidung in den Nieren. Trat der Tod etwas später ein, so war die Blutveränderung entweder noch sehr stark ausgesprochen (Versuch 5) oder abnehmend (Versuch 6 und 7), die Milzschwellung colossal, die Hglb.-Ausscheidung in Nieren und Urin sehr beträchtlich, oder bereits in den Nieren in Abnahme begriffen (Versuch 7).

Ich erlaube mir nun die seit dem Jahre 1879 vorgekommenen Fälle von Vergiftung durch chlorsaure Salze beim Menschen nochmals

1) Virchow's Archiv. 77. Bd. S. 478 .

2) Wiener med. Wochenschrift. 1880 . Nr. $46-48$. 
kurz zusammenzustellen, wobei ich mich auf die genauer beobachteten beschränke. Die meisten sind ohnehin nebst den älteren Beobachtungen in der Arbeit von $v$. Mering angefubrt, aus welcher Stokvis dieselben, wie es scheint, hauptsächlich kennen gelernt hat. Es liegt mir hier besonders an der ïbersichtlichen Gruppirung nach dem Verlaufe.

\section{A. Fälle mit sehr acutem Verlauf; Tod in dem Stadium der schweren Blutveränderung. Dauer einige Slunden bis zu 2 Tagen.}

1. Marchandt), F. Knabe von 6 Jahren. Menge des genossenen Salzes unbekannt. Tod plötzlich in der Nacht. Blut dunkelbraun. Organe ohne Veränderung.

2. v. Haselberg-Hofmeier. ${ }^{2}$ Knabe von $1 \frac{1}{2}$ Jahren. Ein Theelöffel Kali chlor. in Wasser in $1-1 \frac{1}{2}$ Stunden. Plötzlicher Tod am anderen Morgen. Blut braun; Methämoglobin nachgewiesen. Blutkörperchen in der Leiche zum grossen Theil entfärbt. In Milz und Knochenmark reichlich zerfallene Blutkörperchen; keine Nierenaffection.

3. Manouvriez. ${ }^{3}$ ) Mann von 67 Jahren. Nach $35 \mathrm{~g}$ Kali chlor. Erbrechen; Oyanose; Tod nach 8 Stunden. Blut schwarzbraun.

4. Billroth. ${ }^{4}$ ) Mann von 64 Jahren. In 3 Tagen in kleinen Dosen $45 \mathrm{~g}$ Kali chlor. Ziemlich plötzlicher Tod. Blut braun. Nieren angeblich normal. (2 Tage vorher soll der Urin bereits röthlichbraun gewesen sein. - Lithothripsie.)

5. Friedländer.5) Mädchen von 4 Jahren. Tod in wenigen Tagen nach ärztlicher Verordnung von $(4,0) 120,0$ Kali chlor. mehrmals. Plötzlicher Collaps, Erbrechen, Cyanose und gelbbräunliche Hautfarbe. Tod am folgenden Tage. Blut chocoladenbraun, reagirt sauer. Braune körnige Cylinder in den geraden Kanälchen.

6. Zillner. ${ }^{6}$ ) Mann von 31 Jahren. Nach Verbrauch von 11,7 Kali chlor. am 3. Tage Convulsionen und plötzlicher Tod. Hant mit granvioletten Todtenflecken. Blut chocoladenbraun. Nieren ohne Veränderung. Urin in der Blase dunkelgelb.

7. $\mathrm{N}$ e uss.7) Mann von 30 Jahren. $75 \mathrm{~g}$ Kali chlor. irrthümlich innerlich auf einmal genommen. Mehrmals Erbrechen, Cyanose, Athemnoth, Tod nach 6 Stunden. Chocoladenbraune Farbe des Blutes; Milzschwellung, bereits Hämoglobincylinder in den Nieren.

1) Virchow's Archiv. 1879. 77. Bd. S. 456.

2) Berliner klinische Wochenschrift. 1880. Nr. 49.

3) Vgl. Schmidt's Jahrb. 186. Bd. S. 233. 1880.

4) Wiener med. Wochenschrift. 1880. Nr. 44 น. 45.

5) Vgl. Riess, Berliner klinische Wochenschrift. 1882. Nr. 52.

6) Wiener med. Wochenschrift. 1882. Nr. 45 (von Stokvis nach v. Mering fälschlich als Tillner citirt).

7) Deutsche med. Wochenschrift. 1884. S. 57. 
8. Otto. 1) Syphilitischer Mann von 25 Jahren. Plötzlicher Tod in der Nacht; am Tage vorher noch gesund. Die Menge des genossenen Salzes unbekannt (hatte wegen Stomatitis mercur. mit selbst bereiteter Lösung von Kali chlor. gegurgelt). Bhut und Organe von chocoladenbrauner Farbe, Milz stark vergrössert, Pulpa blutreich. Nierensubstanz schmutzig braunroth.

Hieran reiht sich ein neuer von Maschka (Wiener med. Wochenschrift. 1886. Nr. 15) mitgetheilter Fall, in welchem der Tod eines 28 jahl. rigen Mannes wenige Stunden nach irrthümlicher Darreichung von $4 \mathrm{Kaffee-}$ löffeln voll gepulvertem Kali chlor., welche in Wasser gelöst in Zeit von 6 Stunden gegeben waren, unter Durchfällen, Schweiss, Contractionen der Extremitäten und Bewusstlosigkeit erfolgte. Todtenflecke gran. Blut schmutzig braun. In den Nieren bereits Verstopfung der Harnkanälchen. - Die Berliner National-Zeitung vom 28. August d. J. berichtet tiber eine anscheinend ganz acute tödtliche Vergiftung durch chlorsaures Kali.

B. Fälle von weniger acutem Verlauf; die Blutveränderung ist z. Z. des Todes nicht mehr deutlich ausgesprochen, dagegen starke Nierenaffection. Dauer 2-5 Tage.

9. Hofmeie $x^{2}$ )-Brandstäter. ${ }^{3}$ ) Frau von 28 Jahren. In $11 / 2$ Tagen etwa $40 \mathrm{~g}$ Kali chlor. Tod nach 4 Tagen. Milzschwellung; Nieren mit Hglb.-Cylindern vollgestopft.

10. L a nge r. ${ }^{4}$ ) Mann von 21 Jahren. Wegen Halsschmerzen Gurgelungen mit Kali chlor., dabei häufig ein Kaffeelöffel voll verschluckt. Anfang der Intoxication nicht bestimmt; am 4. Tage nach Beginn des Gebrauches aufgenommen mit Cyanose, mässiger Dyspnoe, vollständiger Anurie. Tod ziemlich plötzlich am Abend. Section am folgenden Tage. Blut schmutzig bräunlich. Milz aufs Doppelte vergrössert; Nieren braunroth (mikroskopische Untersuchung nicht angegeben). In der Harnblase wenige Cubikcentimeter triben Urins. Im Blutspectrum Streifen im Roth. Blutkörperchen nicht auffallend verändert, sehr blass.

11. Satlow.5) Knabe von 15 Jahren. In 36 Stunden circa $25 \mathrm{~g}$ Kali chlor. genommen. Erbrechen, Durehfall, Icterus, Cyanose. Urin spärlich, reichliche Hglb.-Cylinder, fast vollständige Anurie, vorïbergehende Besserung nach zweimaliger Transfusion. Tod am 3. Tage. Milz gross, braun; Harnkanälchen mit Hglb.-Cylindern vollgestopft.

12. Bohn. ${ }^{6}$ ) Mann von 50 Jahren. In 36 Stunden $60 \mathrm{~g}$ Kali chlor. Tod nach 2 Tagen. Milz stark geschwollen, braun; Blut nicht deutlich braun. Harnkanälchen ganz mit Hglb.-Cylindern vollgestopft.

1) Petersburger med. Wochenschrift. Nr. 27. S. 235. 1882.

2) Inaugural-Dissertation. Berlin 1880.

3) Deutsche med. Wochenschrift. Nr. 38-40. 1880.

4) Wiener med. Jahrbücher. Jahrgang 1881. S. 473.

5) Jahrbuch für Kinderheilkunde. 1882. N. F. XVII. Bd. S. 311.

6) Deutsche med. Wochenschrift. 1883. Nr. 33. 
13. Goldschmidt.1) Knabe von 3 Wochen. Mund mit feuchtem Lappen mit aufgestreutem Kali chlor. ausgewischt, im Ganzen $4 \mathrm{~g}$ verbraucht, höchstens wohl $1 \mathrm{~g}$ verschluckt. Tief dunkelblauschwarze Verfärbung der Hautdecken, Urin tintenschwarz, allmählich heller werdend. Tod nach 3 Tagen. Keine Section.

14. Leichtenstern. ${ }^{2}$ ) Mann von 33 Jahren. Aus Versehen $25 \mathrm{~g}$ Kali chlor. genommen. Tod nach 5 Tagen; Anurie; alle Symptome der Kali chlor.-Vergiftung.

\section{Chronische Vergiftung mit acutem Endstadium.}

15. Wilke.3) Mann von ca. 50 Jahren. Hatte 4 Wochen sehr anhaltend mit grossen Mengen Kali chlor. gegurgelt; seit 14 Tagen blass, unwohl, der Urin soll schwärzlich gewesen sein. Dann plötzlich mit Erbrechen, Bewusstlosigkeit und Collaps erkrankt. Urin schwarz, trübe. Tod in der folgenden Nacht. Im Urin massenhafte bräunliche Conglomerate, welche auch die gesammten Harnkanälchen ausfüllten.

In diesem Falle werden zwar die bräunlichen Massen als aus veränderten Blutkörperchen bezeichnet, doch stimmt die Beschreibung so genau mit dem gewöhnlichen Verhalten der Hglb.-Massen, dass an der Identität nicht $\mathrm{zu}$ zweifeln ist. Von besonderem Interesse ist hier das vorausgehende chronische Vergiftungsstadium, an welches sich erst die acute Verschlimmerung anschloss.

D. Fälle mit etwas protrahirtem Verlauf; der Tod tritt nach Ablauf der eigentlichen Hämoglobinausscheidung wesentlich infolge der Anurie ein. Dauer 6-12 Tage.

16. Marchand.4) Fall 3. Knabe von 3 Jahren. Etwa $12 \mathrm{~g}$ Kali chlor. in 36 Stunden. Urin nur anfangs dunkel, später gelb, aber äusserst sparsam. Tod am 12. Tage unter Gehirnerscheinungen. In den geraden Kanälchen noch reichliche Hglb.-Cylinder.

17. Wegscheider.5) Mann von 25 Jahren. $40-50 \mathrm{~g}$ Kali chlor. in einigen Stunden. Tod nach 12 Tagen, nachdem die Hglb.-Ausscheidung vortibergegangen war. In den Nierenkanälchen bräunliche cylindrische Massen.

18. Brenner. ${ }^{6}$ ) Mann von 38 Jahren, hatte $30 \mathrm{~g}$ Kali chlor. in Lösung grösstentheils innerlich genommen. Collaps, Cyanose, Erbrechen, Durchfall, schwarzer Urin. Milzschwellung, dann fast vollständige An-

1) Breslauer ärztliche Zeitschrift. 1883. Nr. 1. S. 6.

2) Deutsche med. Wochenschrift. 1884. Nr. 4. S. 89.

3) Berliner klinische Wochenschrift. 1885. Nr. 16 und E. Weinert, Zur Casuistik der Vergiftungen mit chlorsaurem Kali (fleissige aber leider nicht sehr übersichtliche Zusammenstellung von 64 Vergiftungsfällen durch chlorsaures Kali).

4) 1. c. S. 458 .

5) Deutsche med. Wochenschrift. 1880. Nr. 40.

6) Wiener med. Wochenschrift. 1880. Nr. 46-48. 
urie. Am 6. Tage Sopor, klonisehe Krämpfe, plötzlicher Tod. Keine Section.

19. Riess. ${ }^{1)}$ Mann von 36 Jahren. Circa $30 \mathrm{~g}$ Kali chlor. Urin stark vermindert, nur wenige Tropfen von dunkelbrauner Farbe; fast vollständige Anurie bis zu dem $61 / 2$ Tage nach der Vergiftung erfolgenden Tode. Starker Zerfall der rothen Blutkörperchen bis 2 Tage vor dem Tode beobachtet. Sämmtliche Kanälchen der Niere mit Hglb.-Cylindern geftillt.

20. Broesicke und Schadewald. ${ }^{2}$ Mann von 22 Jahren. In 9 Tagen $1 / 4$ Pfund Kali chlor. zum Gurgeln verbraucht. Erbrechen, Cyanose, Icterus, Milzsehwellung, Starrheit der Extremitäten. Fast vollkommene Anurie bis zu dem am 10. Tage erfolgten Tode. Section: Milz geschwollen, gerade und gewundene Harnkanälchen mit $\mathrm{Hglb.} \cdot \mathrm{Cy}-$ lindern vollgestopft.

21. Lingen. ${ }^{3}$ Mann von 18 Jahren. Unbestimmte Menge Kali chlor. durch Vèrschlucken beim Gurgeln genossen. Plötzliche Erkrankung, Collaps, Cyanose, Erbrechen, Durchfall. Urin stark vermindert, trübe braunroth, allmählich heller, mit Sediment ans Hglb.-Cylindern; zunehmende Schwäche, Tod. Keine Section.

\section{E. Geheilte Fälle.}

22. Marchand.4) Fall 2. Knabe von 3 Jahren in 20 Stunden ca. $10 \mathrm{~g}$ Kali chlor. Starke Hglb.-Ausscheidung; Genesung in 14 Tagen.

23. Gesenius. ${ }^{5}$ ) Mann von 25 Jahren. In 13 Stunden $46 \mathrm{~g}$ Kali chlor. Erbrechen, Hämoglobinurie. Heilung nach 7 Tagen.

24. Leichtenstern. ${ }^{6}$ ) Mann von 48 Jahren. Circa $30 \mathrm{~g}$ Kali chlor. Plötzlicher Collaps, starke Hämoglobinurie, dann vollständige Anurie während 7 Tagen. Beginnende urämische Symptome. Am 8. Tage etwas Urin mit braunen körnigen Sedimenten, allmählich heller werdend.

25. Leichtenstern. ${ }^{7}$ Mädchen von 7 Jahren. Hatte ungefähr $20-30 \mathrm{~g}$ Kali chlor. (in welcher Zeit ?) genommen. Plötzlicher Collaps, Blässe, Cyanose; Harnsecretion sistirt; der später gelassene Harn ist klar, frei von Eiweiss.

Leichtenstern weist darauf hin, dass die Intoxication ganz ohne Hglb.-Ausscheidung verlaufen könne. Ob dieselbe auch im Anfang vollständig gefehlt hat, ist wohl nicht sicher zu constatiren gewesen; dass das spätere Auftreten eines gelben Urins mit dem Vorhandensein reichlicher älterer Hglb.-Cylinder in den Sammelröhren der Niere nicht unvereinbar ist, geht aus meinem Fall 3 und Fall 17 hervor.

1) Berliner klinische Wochenschrift. 1882. Nr. 52.

2) Ebenda. 1883. Nr. 42.

3) Petersburger med. Wochenschrift. 1882. Nr.10. (Von Stokvis irrthümlich nach v. Mering als Singer citirt.)

4) 1. c. S. 457 .

5) Deutsche med. Wochenschrift. 1882. Nr. 38. S. 512.

6) Ebenda. 1884. Nr. 4. S. 59.

7) Ebenda. 1884. Nr. 20. S. 305. 
Ich glaube, diese zahlreichen, meist tödtlich verlaufenen Vergiftungsfälle bedürfen kaum der Erläuterung. Sie entsprechen eben den Thierversuchen vollkommen und ergänzen dieselben in mancher Beziehung noch.

Eine ganz bestimmte Abgrenzung der einzelnen Gruppen lässt sich selbstverständlich nicht durchftihren, weder nach den Symptomen, noch nach der Zeitdauer, denn beide werden durch die Art der Aufnahme des Giftes, die Quantität desselben und durch andere Umstände beeinflusst. Im Grossen und Ganzen kann man jedoch diejenigen sehr acut verlaufenden Fälle ohne Zwang als zusammengehörig betrachten, in welchen der Tod im Stadium der ,primären Blutveränderung" eintrat, sodann diejenigen, bei welchen sich bereits eine schwere Nierenaffection entwickelt hatte, während die Alteration des Blutes im Abnehmen war, endlich diejenigen, bei welchen auch die Ausscheidung des gelösten und veränderten Blutfarbestoffes beendet war, und der Tod an den Folgen theils der Anurie durch Verstopfung der Harnkanälchen, theils der Anämie und Entkräftung eintrat. Dass eine Heilung in diesem Stadium selbst nach sehr bedrohlichen Symptomen erfolgen kann, wenn die normale Urinsecretion wieder in Gang kommt, lehrt besonders die interessante Beobachtung von Leichtenstern (24).

Ich gehe nunmehr auf einige Einzelheiten über, zunächst die Betrachtung der Blutveränderung.

Stokvis behauptet, dass die Methämoglobinbildung nach der Einführung chlorsaurer Salze nicht im lebenden, sondern erst im $a b$ gestorbenen Blute eintritt, ja er fasst sogar die Vorgänge der Blutzersetzung unter dem Einfluss der chlorsauren Salze ausserhalb des Organismus als Füulnissvorgänge auf (S. 202).

Diese letztere Bezeichnung ist mindestens ungenau, da es sich, wie anch Stokvis zugiebt, um eine Veränderung handelt, welche sich in derselben Weise in dem Blut unmittelbar nach erfolgtem Tode findet, also zu einer Zeit, in welcher sich Fäulnissvorgänge uberhaupt nicht entwickeln können, noch dazu bei Abwesenheit aller Fäulnissorganismen. Ebensowenig handelt es sich aber auch in einer Blutprobe, welche soeben den Gefässen des lebenden Thieres entnommen und mit chlorsauren Salzen versetzt wird, um eigentliche Fäulniss; ich habe vielmehr schon gezeigt, dass das mit chlorsaurem Kali stärker versetzte Blut auch nach Wochen fast keine faulige Zersetzung zeigt. In ganz frischem, noch lebenswarmem Leichenbiut kann es sich jedenfalls nur um einfache chemische Zersetzungsvorgänge handeln, aber nicht um Fäulniss. Aber gleichviel, ob Fäulniss 
oder einfache Zersetzung - es handelt sich hier vielleicht nur um einen ungenauen Ausdruck -, die Hauptfrage ist, ob eine solche Zersetzung schon im circulirenden Blute bei Anwesenheit chlorsaurer Salze vorkommt, oder ob sie thatsächlich erst am Leichenblute eintritt.

Ich habe gezeigt, dass nach Einfuhrung des Salzes in die Bauchhöhle das während des Lebens aus der Arterie strömende Blut fast von Minute zu Minute eine dunkler werdende Farbe zeigt, dass es die Eigenschaft verliert, sich an der Luft heller zu röthen, dass ferner die Blutprobe, sofort unter dem Spectroskop untersucht, den Methämoglobinstreifen zeigt, während die Streifen des 0-Hglb. schwächer werden. Das Thier stirbt, und das gesammte Blut zeigt sofort dieselbe dunkelbraune Färbung. Stokvis giebt zwar zu, dass einer meiner Versuche für die Richtigkeit meiner Behauptung zu sprechen scheint (S. 193). (In einem zweiten, welchen Stokvis nicht citirt, war die spectroskopische Untersuchung allerdings erst etwas später vorgenommen worden, aber die so augenfällige dunkle Färbung des ausfliessenden Blutes war ganz zweifellos.) Bei näherer Betrachtung beweise jedoch das Auffinden des Methämoglobins in einer Blutprobe unmittelbar vor dem Tode nicht viel, ,erstens nicht, weil wir wissen, dass der Tod nie mit einem Male eintritt und alle Gewebe zu gleicher Zeit trifft, so dass also kurz vor dem Tode das Blut eigentlich nicht mehr als recht lebend betrachtet werden kann. Zweitens nicht, weil es vollkommen unmöglich ist, lebendes Blut dem Körper zu entnehmen, da es ja in demselben Augenblicke des Entnehmens abstirbt" (S. 194).

Ueber den letzteren Punkt liesse sich noch streiten; es ist gewiss richtig, dass das Leichenblut, ja sogar das den Gefässen frisch entnommene Blut nicht ohne Weiteres mit circulirendem Blute identificirt werden darf, dass das Blut aber in demselben Augenblick des Entnehmens abstirbt, dürfte schwerlich zu beweisen sein, namentlich für die rothen Blutkörperchen als solche. Ich persönlich habe von der Lebensfähigkeit derselben eine bessere Meinung. Dass nun aber auch das Blut kurz vor dem Tode in den pulsirenden Arterien - und dieselben pulsiren in solchen Fällen noch recht kräftig, eine mehr and mehr zunehmende Herzlähmung ist vorläufig noch nicht zu constatiren -, dass es auch in diesen nicht mehr "als recht lebend" betrachtet werden kann, das ist doch eine etwas gewagte Behauptung. Gewiss ist das Blut in solchem Falle nicht normal, es ist krank und dem Zerfall geweiht, wenn die Veränderung einen gewissen Grad überschreitet und das Thier dennoch am Leben bleibt. Woran stirbt das Thier anders als an der fortschreitenden Verände- 
rung des Blutes? Es handelt sich eben um eine Veränderung im lebenden Blute, d. h. um eine pathologische und keine cadaveröse Erscheinung, genau wie bei der analogen Veränderung durch Nitrobenzol, Nitrirte u. A., oder wie bei der Bildung des Kohlenoxydund Stickoxydblutes. Auf Seite 195 sagt $S$ t o $k \mathrm{~V}$ is ferner, dass in jenen Versuchen "die Methämoglobinbildung nur in den Augenblicken, in welchen das Blut aus der Ader floss, also nur in dem der Circulation entzogenen Blute, aber hier dann auch sofort, vor sich ging". Wie man sich eine so momentane Einwirkung vorstellen soll, ist mir nicht verständlich; mindestens müsste man doch einen allmählichen Uebergang der Farbe beim Ausströmen beobachten, wovon gar nicht die Rede ist, denn das Blut fliesst eben dunkelbraun aus der Arterie. In sonderbarem Widerspruch zu dieser Auffassung Stokvis' steht die Angabe (S. 187), dass es bei einem künstlichen Chloratzusatz zum Blut bis zu 10 Proc. „bei einer Temperatur von $37^{\circ} \mathrm{C}$. immer eine oder mehrere Stunden dauert, bis die charakteristische braunschwarze Färbung des Blutes eintritt und der Methämoglobinstreifen spectroskopisch erkennbar ist". Auch ich kann das nur bestätigen.

Einen gewissen Schein von Berechtigung könnte jene Behauptung S tok vis' haben, wenn es sich um directe intravenöse Injection des Salzes handelte, da man hier ein Absterben unter dem unmittelbaren Einfluss des Mittels annehmen könnte, welches bei der Resorption nach innerlicher Einverleibung nicht einzutreten brauchte. Wenn man Schwefelsäure ins Blut injicirt, so kann man leicht das Blut innerhalb der Gefässe zum Absterben bringen, aber es würde falsch sein, wenn man aus dieser rein chemisch-physikalischen Wirkung auf die physiologische Wirkung nach der Resorption vom Magen aus schliessen wollte. Beide können identisch sein, wenn nämlich die resorbirte Substanz als solche in das Blut gelangt und hier ihre physikalisch-chemische Wirkung entfalten kann. Das ist nun gerade bei den chlorsauren Salzen der Fall, und darauf beruht, wie ich behaupte, ihre toxische Wirkung. Man kann daher zweifellos die gleiche Wirkung auch durch directe Injection in die Gefässe hervorrufen (wie Stokvis selbst gezeigt hat), aber das scheint mir von untergeordneter Bedeutung, denn es würde zunächst nur beweisen, dass innerhalb der Gefässe dasselbe vor sich gehen kann, wie am Blut ausserhalb des Körpers. Ich glaube daher, dass ich vollkommen berechtigt war, von der intravenösen Injection abzusehen, anstatt, wie Stokvis verlangt, "die Erfolge zu studiren, welche die directe Einfuhr der chlorsauren Salze in das lebende Blut mit 
sich bringt. "Auch die Arbeit 7. Mering's", fährt Stokvis fort, „bringt in dieser Beziehung nur Enttäuschungen, weil alle seine Versuche mit directer intravenöser Injection des Chlorats misslangen" (S. 193) - d. h. bei einem Hunde erhielt ich schnell tödtende Gerinnung im Herzen, bei einem zweiten war die injicirte Menge zu gering gewesen, um eine tödtliche Vergiftung zu erzeugen (1. c. S. 469). Viel wichtiger war es, festzustellen, dass dieselbe Wirkung auf das Blut auch nach der Resorption vom Magen aus eintritt; nachdem dies geschehen und dadurch festgestellt ist, dass das Salz hierbei in gleicher Weise wirkt, kann man selbstverständlich zur Beaniwortung bestimmter Fragen die directe Injection in das Blut vornehmen. Aber diese hat immer etwas Missliches, da bei geringer Concentration grosse Mengen Flüssigkeit erforderlich sind und concentrirte Lösungen andere Nebenwirkungen haben.

Selbstverständlich dauert die Einwirkung der chlorsauren Salze auf das Blut nach dem Tode noch an und eine erst längere Zeit nachher constatirte Braunfärbung könnte sich auch dadurch erklären, dass zur Zeit des Todes noch eine grössere Menge des Salzes im Blute vorhanden war. Andererseits bin auch ich nicht der Meinung, dass die dunkle sepiabraune Farbe ${ }^{1}$ ) des Blutes längere Zeit vor dem Tode bestehen kann, denn das Leben ist eben mit derselben unvereinbar. Aber es ist doch, abgesehen von dem directen Gegenbeweis durch die Beobachtung, nicht anzunehmen, dass eine so äusserst intensive Veränderung mit einem Schlage im Moment des Todes eintritt, um so weniger, als wir wissen, dass ausserhalb des Körpers bei ziemlich reichlichem Zusatz von chlorsauren Salzen das Blut selbst in der Wärme erst nach einiger Zeit sich allmählich verfärbt.

Stokvis behauptet also: Das Blut verändert sich unter dem Einfluss der chlorsauren Salze, weil das Thier stirbt. Woran das Thier aber in Zeit von 1-1 1/2 Stunden zu Grunde geht, wenn ihm $1-1,5 \mathrm{~g}$ pro Kilo chlorsaures Natron in die Bauchhöhle eingespritzt, etwas später, wenn dasselbe subcutan, und noch etwas später, wenn es innerlich beigebracht wird, diese Frage bleibt unbeantwortet.

Stokvis verlangt an Stelle der Untersuchung des aus den Gefässen entnommenen Blates das Studium desselben innerhalb der lebenden Gefässwände. Ich verweise in dieser Beziehung auf die eigenthtimlich cyanotische, oder richtiger graublaue bis bläulichbraune Färbung, welche besonders die sichtbaren Schleimhäute, theilweise auch die Haut in den Fällen von acuter Intoxication durch chlor-

1) Nicht die geringeren Grade der dunkeln Färbung. 
saure Salze, ebenso wie bei der ganz analogen Blutveränderung bei der Vergiftung durch Nitrobenzol u. s. w. aufs Deutlichste zeigen. Diese Färbung wird eben nur durch die intensive dunkelbraune Farbe des Blutes bedingt, sie kommt und verschwindet mit dieser. Mehrere Beobachter vergleichen das Aussehen der Vergifteten im acuten Stadium mit dem bei algider Cholera (B o h n, L i n g e n), ja bei dem ron Gold s c h mid t beobachteten 3 wöchentlichen Kinde wird die ganze Haut tief dunkelblauschwarz wie eine Pflaume bezeichnet. Keineswegs ist diese eigenthümlich graublaue Färbung als Staungserscheinung aufufassen. Stokvis bespricht die tiefe Cyanose in der Schilderung der Vergiftungserscheinungen beim Menschen nach $\nabla$. Mering ebenfalls, erklärt dieselbe jedoch nebst der hochgradigen Dyspnoe, der Herzschwäche, den Convulsionen „theils als Reflexerscheinungen aus der Gastritis toxica, theils als durch Kaliwirkung verursacht" (S. 21:). Er erwähnt ferner als eine ,scheinbar unerklärte Erscheinung grauviolette Flecken der Hant", welche sich in einem meiner Fälle als "rothfleckiges masernähnliches Exanthem" gefunden haben sollen, ähnlich in denen von Wegscheider und Hofmeier. Das von mir und Anderen in späterem Stadium beobachtete maseruähnliche Exanthem hat allerdings nichts mit jener diffusen blaugrauen oder grauvioletten Färbung zu thun, welche schon in den Anfangsstadien der Vergifiung hervortritt. Jenes rothe Exanthem ist auch meiner Ueberzeugung nach eine rein secundäre Erscheinung, vermuthlich vasomotorischen Ursprunges, und nicht durch die Blutveränderung als solche hervorgerufen.

Da nun der meiner Ansicht nach wichtigste und ich möchte sagen absolut sichere Beweis für das Zustandekommen der Blutveränderung im Leben, nämlich die directe Beobachtung des aus den Gefässen strömenden Blutes, sowie die unmittelbar nach dem Tode vorgefundene Farbenveränderung für $S \mathrm{t} o \mathrm{k}$ i s keine beweisende Kraft hat und auch die durch jene bedingte Verfärbung der Haut und sichtbaren Schleimhäute von ihm nicht in diesem Sinne verwerthet wird, so verlangt Stokvis den indirecten Nachweis der Blutzersetzung in Gestalt der Methämoglobinurie. Bevor ich auf diesen Gegenstand eingehe, beztiglich dessen sich in der Arbeit von Stokvis eine Reihe schwerer Irrthümer vorfindet, sei es gestattet, noch etwas näher auf die Art der hier in Betracht kommenden Blutveränderung und ihre Beziehung zur Hämoglobinurie einzugehen.

Bereits bei anderer Gelegenheit ${ }^{1}$ ) ist ausdrücklich hervorgehoben

1) Vgl. Lebedeff, Virchow's Archiv. 91. Bd. S. 271. 
worden, was sich übrigens nach den seitherigen Untersuchungen von selbst ergab, aber doch nicht von allen Seiten richtig aufgefasst worden zu sein scheint, dass die Agentien, welche Hämoglobinurie bervorrufen, sich in zwei vollständig getrennte Gruppen sondern, erstens diejenigen, welche die rothen Blutkörperchen zerstören, ohne den Blutfarbstoff chemisch zu verändern, und zweitens diejenigen, welche zunächst die Umwandlung des Blutfarbstoffes in Methämoglobin bewirken. Zu der ersten Gruppe gehört die Infusion fremden und gelösten Blutes, Verbrennung, Glycerin, das Morchelgift und viele andere. Dahin gehört ferner die paroxysmale Hämoglobinurie. Zu den Agentien der zweiten Art gehören die chlorsauren Salze, die Nitrite und andere. Eigenthümlich verhält sich das Jod im Organismus. Während dasselbe, wie ich gezeigt habe, Hämoglobin ausserhalb des Körpers in sehr kurzer Zeit in Methämoglobin umwandelt, bringt dasselbe bei Einverleibung in den Körper zwar starke Hämoglobinausscheidung infolge der Lösung der rothen Blutkörperchen hervor, aber nicht die eigenthümliche Blutfärbung durch Methämoglobin.

Die histologischen Veränderungen der rothen Blutkörperchen sind in beiden Reihen wesentlich dieselben: Lösung (Extraction) des Blutfarbstoffes und Zerfall der rothen Blutkörperchen in kleine Bruchstiicke. Allerdings wirken die einen Agentien mehr in dieser, die anderen mehr in jener Weise. Während destillirtes Wasser, Glycerin und andere wesentlich eine Lösung des gefärbten Bestandtheiles und schliesslich der ganzen Körperchen herbeiführen, bringen andere mehr die Abschnürung kleiner gefärbter Partikel und vollständigen Zerfall in Körnchen hervor, wobei jedoch ebenfalls stets ein Theil dès Farbstoffes in Lösung geht. Diese Veränderung tritt bekanntlich am deutlichsten bei künstlicher Erhitzung des Blutes ein (M. Schultze), und ebenso auch ganz besonders bei der Verbrennung oder Verbrühung am lebenden Körper (Ponfick). Aehnlich verbält sich der Zerfall bei Vergiftung durch Toluylendiamin (A fanassiew).

Findet der Zerfall sehr acut und in grosser Ausdehnung statt, wie bei schweren Verbrennungen, so gelingt es leicht, die zerstörten rothen Blutkörperchen im circulirenden Blute nachzuweisen (Ponfick), aber diese Erscheinung geht in der Regel schnell voriber, weil das Blut sich sehr bald seiner körnigen Zerfallsproducte durch Anhäufung in der Milz entledigt. Eine Umwandlung in Methämoglobin findet in diesen Fällen nicht statt, wenigstens kommt niemals weder die eigenthümliche chocoladenbraune Farbe des Blutes und der Organe, noch der Methämoglobinstreifen im.Spectrum zur Beob- 
achtung. Ich kann daher vorläufig die Ansicht Ponfick's'), dass das Methämoglobin gewissermaassen eine Zwischenstufe zwischen der Umwandlung des Hämoglobin in Bilirubin darstellt, noch nicht als erwiesen betrachten. Für die Behauptung Ehrlich's ${ }^{2}$ ), dass das gelöste Hämoglobin sich stets im circulirenden Blute sofort in Methämoglobin unwandele, scheint mir kein Anhaltspunkt vorzuliegen, denn das Blut bleibt in solchen Fällen immer roth, wenn es nicht vorher bereits die charakteristische Umwandlung erlitten hatte.

Aber es ist wohl als sicher zu betrachten, dass die zerfallenen Blutbestandtheile in der Milz weiter verarbeitet und der Leber zugeführt werden, während ein Theil in der Milz als eisenhaltiges Pigment iubrig bleibt. $\mathrm{Ob}$ die Leberzellen auch an und für sich im Stande sind, die zerfallenen Reste aus dem Blutstrom direct aufzunehmen und weiter za verarbeiten, oder ob die Milz dabei immer als Zwischenstufe nothwendig ist, dürfte wohl noch nicht sicher zu entscheiden sein. Die zuweilen sehr reichliche Anhäufung eisenhaltiger Pigmente, welche auch in der Leber unter analogen Verbältnissen nicht selten gefunden wird, wie namentlich Quincke gezeigt hat, scheint mir noch nicht ohne Weiteres für jene Annahme zu sprechen.

Thatsache ist aber, dass in allen Fällen, in welchen ein reichlicher Untergang von rothen Blutkörperchen stattfindet, eine Hypercholie, Vermehrung der gefärbten Bestandtheile, nicht selten auch Icterus (welchen man vielleicht als Resorptionsicterus im Gegensatz zu Retentionsicterus bezeichnen könnte) vorkommt.

Der Ablauf der histologischen Veränderungen des Blutes unter dem Einfluss der chlorsauren Salze ist noch nicht hinlänglich verfolgt. Ich behaupte aber auch jetzt noch, was ich bereits 1879 angegeben habe, dass in dem Stadium der ,primären Blutveränderung"; (der ,Methämoglobinämie") noch keine bemerkenswerthe histologische Veränderung der rothen Blutkörperchen (vielleicht abgesehen von einer geringen Quellung derselben) eintritt, weder ein Zerfall in Körnchen, noch eine Auflösung. Der Tod kann dabei also lediglich die

1) Verhandlungen des Congresses für innere Medicin. Wiesbaden 1883. S. 212 .

2) Charité-Annalen. 1885. S. 136 und Deutsche medicinische Wochenschrift 1881. S.225. Die an letzterer Stelle mitgetheilte Beobachtung von paroxysmaler Hămoglobinurie bei einer Syphilitischen, bei welcher im Urin nur Methämoglobin nachzuweisen war, ist von dem gewöhnlichen Verhalten durchaus abweichend. Möglicherweise lag hier eine Complication vor. Es fehlt aber auch der Nachweis des Methämoglobin im Blute. 
Folge der chemischen Umwandlung des Oxyhämoglobin sein, wenn man nicht etwa neben dieser handgreiflichen Veränderung es vorzieht, zu einer davon unabhängigen hypothetischen Einwirkung auf das Centralnervensystem oder das Herz seine Zuflucht zu nehmen, fur welche - wie ich kaum zu bemerken brauche - nicht der geringste Anhaltspunkt vorliegt.

Tritt der Tod in diesem Stadium noch nicht ein, so werden die rothen Blutkörperchen zerstört und der Grad der Zerstörung hängt augenscheinlich von der Schwere der Vergiftung ab. Ich verweise in dieser Beziehung auf einen meiner ersten Versuche: ,sehr dünne Schichten Blut, direct aus dem Herzen entnommen, zeigen sehr zahlreiche, ganz farblose, zum Theil verkleinerte Ringe, offenbar Blutkörperchen, welche ihren Farbstoff abgegeben haben ". ${ }^{1)}$ Allerdings fand die Untersuchung des Blutes nicht im Leben, sondern erst kurze Zeit nach dem Tode statt (höchstens 1-2 Stunden), aber das Blut war noch vollständig dünnflüssig. Auch kann ich hinzufügen, dass man diese Erscheinung bei der ganz acuten Intoxication auch mehrere Stunden nach dem Tode noch nicht antrifft, sondern nur dann, wenn bereits Zeichen des Zerfalles der rothen Blutkörperchen im Leben eingetreten waren, was in jenem Versuche der Fall war. Auch findet man jene Veränderung der rothen Blutkörperchen nìcht so bald, wenn man ausserhalb des Organismus Blut mit chlorsauren Salzen mischt. Es ist also keine Leichenerscheinung, sondern ebenfalls eine pathologische Veränderung, welehe sich eine gewisse Zeit nach dem Eintritt der Umwandlung des Blutfarbstoffes im circulirenden Blute einstellt.

Jedenfalls beginnt der Zerfall der rothen Blutkörperchen sehr bald, was aus der schnellen Entstehung des Milztumor und dem frühzeitigen Eintritt der Hämoglobinausscheidung durch die Nieren hervorgeht. Ein Blick auf die obigen Versuche lehrt, dass die Milzschwellung bereits einen sehr hohen Grad erreichen kann, bevor die Ausscheidung des gelösten Farbstoffes durch die Nieren beginnt, oder wenigstens deutlich nachweisbar wird. Was die erstere betrifft, so kann dieselbe in der That schon im Laufe weniger Stunden bis zu einem Tage eine geradezu colossale Grösse erreichen (s. oben Versuch 3, 5, 7); die Kapsel der Milz ist aufs Aeusserste gespannt. Die mikroskopische Untersuchung der schwarzbraunen Milzpulpa weist neben eigenthümlich zusammengeballten rothen Blutkörperchen auch massenhafte Zerfallsproducte von solchen in allen Formen, besonders

1) Virchow's Archiv. 77. Bd. S. 470. 
freie Körnchen, aber auch rothe Blutkörperchen in sehr verschiedener Gestalt und in verschiedenen Stadien der Entfärbung nach. ${ }^{1}$ ) Daraus geht hervor, dass auch bei der Intoxication durch chlorsaure Salze, wie bei anderen Ursachen der Zerstörung der rothen Blutkörperchen, ausser einer einfachen Lösung auch ein körniger Zerfall derselben eintritt, und zwar ist es sicher, dass dieser an den zu Grunde gegangenen und in der Milz angehäuften Körperchen noch weitere Fortschritte macht.

Der Milztumor entspricht somit, abgesehen von seiner eigenthïmlich dunkelbraunen Farbe, genau demjenigen, welcher auch bei anderen Formen schwerer Hämoglobinurie mit Zerfall der rothen Blutkörperchen vorkommt, während derselbe bei einfacher Lösung der Blutkörperchen durch Glycerin, destillirtes Wasser u. s. w. vollkommen fehlen kann, oder nur geringfügig ist.

Stokvis erblickt freilich in diesem nicht selten enormen Milztumor, der eine so höchst charakteristische Beschaffenheit besitzt - einer Theorie zu Liebe - nichts Anderes als eine Milzschwellung infolge von katarrhalischem Icterus, welcher selbst von einer Gastroenteritis herrubren soll! Abgesehen davon, dass zur Zeit des Eintrittes der Milzschwellung noch gar kein Icterus vorhanden zu sein pflegt, ist die erstere ganz anders beschaffen und sehr viel bedeutender, als der Milztumor bei Icterus, der, wenn er überhaupt constant vorkommen sollte, ziemlich geringfügig sein dürfte. Die Versuche, welche $\mathrm{Mackay^{2 }}$ ) unter Stok vis' Leitung in dieser Richtung angestellt hat, haben kein sehr befriedigendes Ergebniss gehabt.

An dieser Stelle muss ich den von Riess veröffentlichten Fall von Vergiftung durch chlorsaures Kali erwähnen (s. oben Fall 19), da Riess das Hauptgewicht bei demselben auf den Nachweis einer mikroskopischen Blutveränderung legt. „Ein grosser Theil (etwa 1/4 bis $1 / 5$ ) der rothen Blutkörperchen ist im Haupttheil seines Stroma vollständig entfärbt und enthält nur Reste seines farbigen Inhaltes in Form kleiner, meist rundlicher hämoglobingefärbter Kügelehen und Körnchen, die in Zahl von $1-5$ in dem farblosen Stroma zerstreut liegen. Dabei sind die, tibrigens scharf contourirten, entfärbten Körperchen meist etwas, einige bedeutend kleiner als die normalen rothen Blutkörperchen und fast sämmtlich von länglicher, eliptischer oder ovoider Form. Aehnliche hämoglobingefärbte Kügelchen, wie in

1) Virchow's Archiv. 77. Bd. S. 470; 91. Bd. S. 276.

2) Dieses Archiv. XIX. Bd. S. 269. 
diesen Körperchen, finden sich auch reichlich zwischen denselben frei im Serum". 1)

Diese Erscheinung wurde in abnehmender Intensität 3 Tage hindurch bis 2 Tage vor dem Tode beobachtet, welcher am 6 . Tage nach der Vergiftung erfolgte. Die Hautfärbung war schon am 2. Tage schmutzig gelbbräunlich, das Blut war sehr dunkel, doch ergab dje spectroskopische Untersuchung nichts Abnormes. (Dies ist an sich nicht auffallend, da die spectroskopisch wahrnehmbare Blutveränderung uberhaupt nicht von sehr langer Dauer za sein pflegt.)

Zweifellos ist diese Beobachtung von grossem Interesse, denn sie zeigt in deutlichster Weise, was in anderen Fällen am Menschen bis dahin vermisst wurde, das Vorbandensein von Zerfallsformen rother Blutkörperchen im circulirenden Blute. Nur ist Riess meiner Ansicht nach im Irrthum, wenn er gerade diesen Befund als besonders charakteristisch für die Intoxication durch chlorsaure Salze ansieht und der Meinung ist, dass diese Veränderung bisher bei keinem pathologischen Processe beobachtet worden ist. Denn, wie erwähnt, wird dieselbe auch in anderen Formen von Hämoglobinurie gefunden und andererseits kann sie gerade bei der tödtlichen Vergiftung durch chlorsaure Salze ganz fehlen. „Klarer, als die frttheren Fälle, zeigt dieser die Art der Veränderung an, welche für die Blutkörperchen unter der Einwirkung des chlorsauren Kalium anzunehmen ist. Während die älteren Mittheilungen nur (?) eine chemische Umwandlung des Hämoglobin oder eine Auflösung des Blutfarbstoffes im Serum nachweisen, resp. supponiren, sehen wir hier eine eigenthtimliche Formveränderung des Blutkörpercheninhaltes unter dem Einfluss des Giftes eintreten." Aus dem oben Gesagten geht hervor, dass Beides, die chemische Umwandlung und der Zerfall der Blutkörperchen durchaus einander nicht ausschliesst, ja im Gegentheil, der letztere eine nothwendige Folge der ersteren ist.

Ferner kann ich Riess nicht beistimmen, wenn er die Ansicht ausspricht, dass gerade der körnige Zerfall der rothen Blutkörperchen eine weitere Erscheinung erklärt, nämlich die Bildung der Blutcylinder in den Nieren, „während bei gleichmässiger Lösung des Blutfarbstoffes im Plasma, nach unseren Kenntnissen über anderweitige hämoglobinurische Processe, eine Bildung körniger, die Harnkanälchen verstopfender und zu letaler Anurie führender Bluteylinder in den Nieren nicht zu erwarten wäre". Das ist irrthümlich, denn genau dieselben körnigen Hglb.-Cylinder kommen in den Nieren und

1) l. c. S. 4 des Separatabdruckes, mit Abbildung. 
im Urin in allen schweren Formen von Hämoglobinurie vor, auch bei denen, bei welchen nur gelöstes Hämoglobin vorhanden ist, und gerade die körnigen Bestandtheile werden nicht durch die Nieren ausgeschieden. Vielmehr entstehen die körnigen und tropfenartigen, den rothen Blutkörperchen manchmal sebr ähnlichen Massen erst durch Gerinnung in den Kanälchen. (Ich selbst habe in meiner mehrfach citirten Arbeit ursprünglich ebenfalls die Vermuthung geäussert, dass die Zerfallsproducte der rothen Blutkörperchen durch eine Art Diagerose in die Harnkanälchen gelangen; ich habe aber später mich von der Richtigkeit der von Pon fick stets betonten Ansicht tiberzeugt, dass es sich um Ausscheidung ursprünglich gelösten Hämoglobins handele.)

Wie erklärt $\mathrm{S}$ tok $\mathrm{v}$ is diesen histologisch so klar nachgewiesenen Zerfall der rothen Blutkörperchen, selbst wenn er die chemische Umwandlung des Blutfarbstoffes während des Lebens nicht anerkennen will? Stokvis citirt allerdings den Fall von Riess, indess, wie es scheint, nicht nach dem Original, sonst wäre es wohl kaum möglich, dass er uber die Vergiftungsfälle beim Menschen äusserte: „Kein einziges Vergiftungssymptom zwingt mit Nothwendigkeit zu der Annahme einer so intensiven Blutveränderung, dass die Erhaltung des Lebens unmöglich wird, und“, fährt er fort, „die äusserst geringe Veränderung der Nieren weist jedenfalls nur auf eine beginnende Reizung dieser Organe hin" (S. 211).

S tok vis betrachtet nun das Nichtauftreten der Hämoglobinurie (oder Methämoglobinurie) in den ganz acut verlaufenden Vergiftungsfällen als einen Hauptbeweis gegen die stattgehabte Blutalteration. Er vermisst die Ausscheidung durch den Urin sowohl in einem meiner Versuche am Hunde (richtiger "in zweien") als in einem analogen von Is ambert und einem von v. Mering, in welchem ein Hund von $10 \mathrm{~kg} 5$ Stunden nach der Einfuhrung von $5 \mathrm{~g}$ Natriumchlorat in die Vena jugularis und vorheriger Unterbindung der Ureteren getödtet wurde, wobei das Blut die charakteristische Verfärbung zeigte. Kann S t o k vis ernstlich annehmen, dass bei einer Blutveränderung, welche in dem einen Falle 9, in dem anderen 65 Minuten vor dem Tode eintrat, bereits eine Ausscheidung von gelöstem Blutfarbstoff durch die Nieren statthaben könne? Ueberdies wurde ausdrücklich hervorgehoben, dass in diesem ersten Stadium der Blutverminderung uberhaupt noch kein Zerfall, keine Lösung der rothen Blutkörperchen nachzuweisen ist. In zwei anderen Fällen konnten wir 3, resp. 5 Stunden nach der Einführung bereits starke Milzschwellung und soeben beginnende Ausscheidung durch die Nieren 
constatiren. ${ }^{1)}$ Offenbar hängt das Eintreten der letzteren von der Art der Einverleibung und besonders anch von der eingeführten Menge des Salzes ab. Auch bei allen anderen Formen von Hämoglobinurie verstreicht zwischen Application des schädlichen Agens, Glycerin, Jod u. s. w. und dem Beginn der Hglb.-Ausscheidung durch die Nieren meist ein Zeitraum von mehreren Stunden.

Dieses Fehlen der Methämoglobinurie in den ganz acut tödtlichen Fällen wird nun von $S$ to $\mathrm{k} v$ is in einer Weise verallgemeinert, welche ich mir, in Anbetracht der mehrfach wiederholten genauen Beschreibung der so höchst charakteristischen Nierenveränderung beim Hunde (und beim Menschen), beim besten Willen nicht erklären kann. „Man muss hier einen Unterschied machen zwischen Hunden und Kaninchen in dem Sinne, dass bei Hunden nie von Hämoglobinurie und Methämoglobinurie die Rede ist, auch wenn so grosse Dosen ins Blut gebracht worden sind, dass deutliche Vergiftungserscheinungen oder der Tod eintraten. Nur Eiweiss wird in kleiner Menge bisweilen vorübergehend im Harn angetroffen" (S. 197). Bei Kaninchen soll sich die bisweilen vorkommende Methämoglobinurie in der Weise erklären, dass durch das durch die Nieren ausgeschiedene Salz in concentrirter Lösung eine starke Reizung der Nieren und dadurch Blutung herbeigeführt wird; durch das gleichzeitig ausgeschiedene chlorsaure Salz werde dann selbverständlich aus dem Hämoglobin des bluthaltigen Harns Methämoglobin gebildet.

Man vergleiche damit die makroskopisehe und mikroskopische Beschreibung der Nieren in den oben angeführten Versuchen 5, 6, 7, sowie die analogen Befunde vom Menschen. Eine nochmalige Wiederholung derselben kann ich hier füglich ersparen, bemerke nur so viel, dass es sich stets und in allen Fällen um eine reine Hämoglobin- (resp. Methämoglobin-)Ausscheidung handelt und nie um $H \ddot{a}-$ maturie. Allerdings sind zahlreiche Verwechselungen zwischen den eigenthümlich körnigen, tropfenartigen, glänzenden Gebilden in Harnkanälchen und Urin mit Blutkörperchen vorgekommen und kommen noch vor, indess ist es nicht schwer, sich von dem ganz verschiedenen Verhalten beider Dinge zu überzeugen. Ueberdies ist der Befund der Niere bei der Intoxication durch chlorsaure Salze histologisch durchaus identisch mit dem aller anderen Hämoglobinnieren, was mir hinreichend aus eigener Anschaung bekannt ist und ebenso aus den zahlreichen Beschreibungen von Ponfick, Boström, Lebedeff,

1) $\mathrm{S}$. Versuch 3 und 4. Allerdings waren in letzterem bereits kleinere Dosen der letalen vorausgegangen, in ersterem fand die Einführung selbst in $3 \frac{1 / 2}{2}$ Stunden statt. 
Afanassiew und Anderen klar genug hervorgeht. Der Ausdruck "Blutkörpercheninfarct" der Harnkanälchen, welchen A ckermann neuerdings anf die Niere bei Kali chloricum-Vergiftung anwendet (vgl. Fall Wilke) ist demnach nicht ganz zutreffend. Es ist nicht undenkbar, dass bei dieser schweren Affection, welche natürlich Circulationsstörungen in der Niere verursacht, auch gelegentlich Blutkörperchen als solche austreten können, indess würden diese an dem Charakter der ganzen Affection gar nichts ändern.

Ich bin überzeugt, dass auch Stokvis, wenn er einmal eine solche Niere eines Hundes nach Vergiftung durch chlorsaures Kali oder Natron gesehen haben wird, welche in allen Abschnitten des Kanälchensystems mit den charakteristisehen homogenen röthlichbraunen Cylindern, sowie mit den glänzenden körnigen oder tropfenähnlichen Conglomeraten gefüllt ist, den Gedanken aufgeben wird, dass es sich nur um eine geringfügige locale Reizung der Nieren durch das ausgeschiedene Salz handelt.

Ich gehe hier nicht auf die Frage ein, ob die Nierenaffection bei der Hämoglobinurie und Methämoglobinurie als eine Nephritis im gewöhnlichen Sinne aufzufassen ist oder nicht, indem ich in dieser Beziehung auf die Arbeit von Lebedeff, Afan assiew und Anderer verweise. Meiner Ueberzeugung nach handelt es sich um einen einfachen Ausscheidungsvorgang und keine Entztindung, denn im frischen Stadium fehlen alle wirklichen entzündlichen Veränderungen. Es macht für die Niere keinen Unterschied, ob die bräunlichen Massen des Hämoglobins oder die blauen des indigschwefelsauren Natrons oder die gelben und grïnen des Gallenfarbstoffes ausgeschieden werden. Damit ist jedoch nicht ausgeschlossen, dass sich im weiteren Verlauf und bei längerer Dauer auch Degenerationen des Epithels und entzündliche Veränderungen im Zwischengewebe anschliessen können. In leichten Graden geht die Ausscheidung spurlos vorüber. Dabei sei noch bemerkt, dass die Hämoglobinausscheidung in den Anfangsstadien in den Rindenkanälchen, wahrscheinlich auch zugleich in den Schleifenkanälchen auftritt, dass aber eine Füllung der geraden Kanälchen, besonders des Markes, immer auf eine bereits vorgeschrittene, selbst auf eine bereits beendigte Ausscheidung hindeutet. Ich will hier auch nicht die weiteren Fragen bertibren, ob die Hglb. - Ausscheidung durch die Glomeruli oder durch die Nierenepithelien zu Stande kommt, da dies für die vorliegende Frage unwesentlich ist, ich hoffe aber später auf diesen Gegenstand zurückkommen zu können.

Stokvis stellt also erstens die Umwandlung des Oxyhämoglobin in Methämoglobin im lebenden Blute in Abrede, abwohl dieselbe durch 
einige meiner Versuche positiv bewiesen und durch v. Mering (Versuch 108 S. 125) bestätigt worden ist. Er deutet den Milztumor, der durch Anhäufung der massenhaften Zerfallsproducte rother Blutkörperchen in kürzester Zeit entsteht, als Folge eines Icterus, welcher noch nicht vorhanden ist. Er bestreitet ausdrücklich das Vorkommen der Hämoglobinausscheidung durch die Nieren bei Hunden, obwohl dasselbe durch eine Reihe sorgfältig ausgeführter Untersuchungen nachgewiesen ist und überdies dasjenige Symptom darstell, welches schon bei mässigen Graden der Intoxication am augenfälligsten hervortritt. Alles dies geschieht, ohne dass Stokvis selbst irgend einen Versuch mittheilt, in welchem beim Hunde trotz der Einführung grösserer Dosen des Salzes das Versuchsergebniss ein von dem meinigen abweichendes gewesen ist. Allerdings sagt Stokvis Seite 205: „Das Intoxicationsbild, welches Hunde und Kaninchen bei der intravenösen Injection des Na-Chlorats darbieten, wenn $2-21 / 2 \mathrm{~g}$ pro Kilo Thier injicirt werden, hat alle Hauptzige mit demjenigen gemein, welches auch bei Fröschen hervortritt." Darauf beschreibt er - offenbar hauptsächlich nach Versuchen an Kaninchen - die musculären Zuckungen und Convulsionen, sodann die Erscheinungen der Depression, den komatösen und paralytischen Zustand mit deutlicher Dyspnoe und Störung der Herzaction und Tod unter heftigen Convulsionen nach 1-2 Stunden. „Bei Hunden wird während der Injection eine starke Zunahme der Frequenz, später eine grosse Unregelmässigkeit (der Herzcontractionen) beobachtet." Stokvis hat es dabei, wie es scheint, nicht der Mühe werth erachtet, eine Blutprobe während des Lebens zu untersuchen, wenigstens wird daruber nichts mitgetheilt; sollte er wirklich so von der Ueberzeugung durchdrungen gewesen sein, dass das Blut bei allen diesen Thieren nicht mehr als ,recht lebend" zu betrachten gewesen sei?

Mit welchem Rechte Stokris also die Existenz einer specifischen Giftwirkung der chlorsauren Salze tiberhaupt in Abrede stellt, dies zu entscheiden, glaube ich getrost dem Urtheil des Lesers iiberlassen zu dürfen.

Stokvis sucht nun zu zeigen, dass "die toxische und letale Wirkung des intravenös injicirten Na-Chlorats in allen Stiucken derjenigen concentrirter Kochsalzlösungen ähnlich" sei, und dass auch bei innerlicher Darreichung beide Salze in ihrer Wirkung übereinstimmen (S. 207). Die letale Dosis betrage für beide Substanzen 8-10 g pro Kilo Thier, wobei allerdings der einschränkende Zusatz gemacht wird "wenigstens bei Kaninchen“, da keine Hunde zu diesen Versuchen verwendet wurden, um nicht zu viel Thiere ohne 
dringende Nothwendigkeit zu opfern. Es scheint mir doch, so gern ich auch diesen Grund anerkennen möchte, dass in diesem Falle die Nothwendigkeit vorlag, einige Hunde zu benutzen, wenn Stokvis den Nachweis führen wollte, dass meine früheren Versuchsergebnisse irrthümlich waren. Denn ich hatte bereits gezeigt, wenn ich hier auch von den Versuchen Le be deff's absehe, welche St ok vis nicht berïcksichtigt, dass $2,8 \mathrm{~g}$ pro Kilo in 5 Dosen einen Hund unter Hervorbringung der charakteristisehen Blutveränderung mit intensivster Hämoglobinausscheidung tödteten, und dass $2 \mathrm{~g}$ per Kilo in 3 Dosen die Blutveränderung mit colossaler Milzschwellung und starker Hämoglobinausscheidung hervorbrachten. Von vornherein ist schon anzunehmen, dass bei einmaliger Einfübrung eine weit geringere Menge genügt, um bei innerlicher Darreichung den Tod herbeizufübren.

Die Vergiftungserscheinungen bei letzterer Applicationsart sucht nun Stokvis zum grössten Theil auf die "Gastritis toxica" und die durch die heftige Reizung des Magens bedingten Reflexe auf Respirations- und Herzcentren zurückzuführen. Bezüglich der ersteren kann ich hier nur hervorheben, dass der Magen bei Hunden keine Veränderung darbietet, welche auf eine Gastritis oder gar auf eine Anätzung hindeuteten. In dem einen meiner früheren Versuche, in welchem die relativ colossale Dosis von $50 \mathrm{~g} \mathrm{Na}$. chloric. in 5 Dosen, nach vorhergegangenem mebrtägigen Gebrauch kleinerer Dosen, angewendet war (1. c. S. 470), hatte der Magen einen rauchgrauen Anstrich, einige Stellen im Fundus waren besonders dunkelbräunlich (doch handelte es sich dabei keineswegs um Blutergüsse); in dem 2. Fall (S. 479) wird die Schleimhaut als „blassröthlich, ohne Veränderung" bezeichnet.

Nur in einem Fall von Lebedeff, bei welchem durch mehrere Tage allmählich steigende Dosen des Salzes gegeben wurden, stellte sich am Tage vor dem Tode eine Entleerung blutiger Massen aus dem After ein, und die Darmschleimhaut, besonders die des Dickdarmes, fand sich bei der Section mit blutiger Flüssigkeit bedeckt, jedoch ohne Substanzverluste, welche auch im Magen, der eine helle Fluissigkeit enthielt, fehlten (1. c. S. 276). Ich bin geneigt, auch diese Darmaffection nicht für die directe Folge der Einwirkung des Salzes auf die Darmwand zu halten, sondern für eine secundäre, von der bereits erfolgten Blutveränderung abhängige Erscheinung, welche man in ähnlicher Weise auch bei anderen nicht ätzenden Giften bei Hunden nicht selten antrifft - ich erinnere z. B. an die septische Vergiftung. Ich will keineswegs behaupten, dass eine grosse Menge des 
Salzes bei innerlicher Einführung ganz ohne Wirkung auf die Verdauungsorgane ist; es kann beispielsweise eine Erhöhung der Peristaltik die Folge derselben sein; eine Anätzung der Schleimhaut, eine eigentliche Gastritis tritt indess bei Hunden nicht ein. Das häufig vorkommende Erbrechen ist vielmehr auf den bereits erfolgten Eintritt der Blutveränderung zu beziehen. Uebrigens ist der beste $\mathrm{Be}-$ weis, dass die gastrischen Störungen rein secundärer Natur und keineswegs geeignet sind, den charakteristischen Symptomencomplex der Vergiftung aufzuklären, die einfache Thatsache, dass dieser letztere genau in derselben Weise verläuft, gleichviel ob das Gift per os, oder durch die Peritonealhöhle, oder subcutan, oder anderweitig beigebracht worden ist. - Beim Kaninchen fand S tok vis die „Schleimhaut des Magens immer in hohem Grade angeätzt"; dieselbe zeigte besonders an der grossen Curvatur intensive und ausgedehnte Blutungen, welche sich bis in die Submucosa ausdehnen; ein grösserer oder geringerer Theil der Schleimhaut liess sich wie ein Fetzen von der Unterlage abheben, „mit einem Wort, die der Gastritis toxica zukommenden Erscheinungen in ausgeprägtester Weise."

Wie erklärt nun Stokvis die Vergiftungserscheinungen beim Menschen? Das hartnäckige Erbrechen, die profuse Diarrhoe bei den ganz acut verlaufenden Fällen (in welchen diese Symptome ubrigens gar nicht einmal constant sind) soll sich viel leichter aus der Gastritis toxica nach Einfuhr einer grösseren Menge eines reizenden Salzes erklären, als aus einer Blutzersetzung. „Die hochgradige Dyspnoe, die tiefe Cyanose, die Herzschwäche, die Convulsionen sind weiter theils als Reflexerscheinungen aus der Gastritis toxica, theils als durch Kaliwirkung verursacht zu betrachten, theils auch mit der acuten Wirkung einer in die Blutmasse aufgenommenen concentrirten Salzlösung in Zusammenhang zu bringen"(S. 211). Bei den weniger acuten Fällen ist es ,dieselbe locale Wirkung (scil. welche die toxische Gastritis hervorbringt), welche beim Fortdanern des Lebens auch eine Schwellung der Duodenalschleimhaut veranlassen kann, als deren Folge ein echter Icterus catarrhalis, ein wahrer hepatogener Icterus auftritt. Dieser Icterus führt zur Gallenstauung und zur nothwendigen Volumsvergrösserung der Leber, und ausserdem, wie jeder hepatogene Icterus, zur Schwellung der Milz. Die entfernte Kali- und Salzwirkung äussert sich als Herzschwäche und Athemnoth, die allmähliche Abscheidung des Salzes durch die Nieren, welche bei den eingebrachten grossen Mengen mehrere Tage in Anspruch nimmt, führt ausserdem zu einer acuten toxischen Nephritis, wobei in geringer Menge ein Eiweiss, Blut und Harneylinder enthaltender Harn entleert, 
wird .... - Es entwickeln sich dann die von der Urämie abhängigen Störungen des Nervensystems . . .

Man sieht, wie leicht alle Vergiftungserscheinungen sich erklären lassen und wie wenig Veranlassung da ist, noch irgend ein anderes unbewiesenes Moment (!) zur Erklärung des Vergiftungsbildes heranzuziehen" (S. 213).

Ich würde nur das oben Gesagte wiederholen können, um nachzuweisen, dass fast kein einziger Zug dieses Bildes mit dem Resultat des Experimentes und der anatomischen Untersuchung am Menschen stimmt. Nur den Schlusssatz bezuglich der Urämie vermag ich zu acceptiren, jedoch mit dem Unterschiede, dass sich die Urämie durch die mechanische Verstopfung der Sammelröhren der Nieren (ja sogar des Nierenbeckens und der Blasenmündung) durch die veränderten Hglb.-Massen und die dadurch bedingte Anurie erklärt (s. oben).

Man ist nun wohl zu der Frage berechtigt, wie es zu erklären ist, dass Stokvis trotz einer grossen Anzahl von Thierversuchen zu so abweichenden Ergebnissen gekommen ist, dass er, wie aus seiner Schilderung hervorgeht, das charakteristische Bild der Vergiftung durch chlorsaure Salze in allen ihren Stadien überhaupt nicht zu sehen bekommen hat. Der Grund liegt zum grossen Theil offenbar darin, dass S to $\mathrm{kv}$ is fast ausschliesslich an Kaninchen experimentirte, während ich und Andere hauptsächlich Hunde benutzten. Ich hatte mich bereits im Anfang meiner Versuche überzeugt, dass Kaninchen sich viel widerstandsfähiger gegen chlorsaure Salze verhalten als Hunde. Die Versuche an diesen gaben aber so übereinstimmende Resultate mit den Vergiftungserscheinungen beim Menschen, dass dieselben ftir die Hauptfrage, die Ursache der giftigen Wirkung der chlorsauren Salze auf den menschlichen Organismus, jedenfalls ausschlaggebend sein mussten.

Der Schluss, zu welchem Stokvis aus seinen Versuchen in erster Linie meiner Ansicht nach hätte gelangen müssen, wäre gewesen, dass Kaninchen nicht die geeigneten Versuchsthiere sind, um diese Frage zur Entscheidung zu bringen. Es ist doch eine alte Regel und ein nicht unbilliges Verlangen, dass, wenn Jemand die Unrichtigkeit gewisser Versuche beweisen will, er dieselben zunächst unter denselben Bedingungen wiederholen muss.

Was den Grund des verschiedenen Verhaltens der Versuchsthiere betrifft, so kann man wohl, ganz allgemein gesagt, annehmen, dass der Organismus der Pflanzenfresser sich gegen chlorsaure Salze anders verhält als der der Fleischfresser und des Menschen. Der erstere scheint über Mittel zu gebieten, um den schädlichen Einfluss der chlor- 
sauren Salze bei ihrem Durchgang durch die Blutbahn in höherem Grade zu neutralisiren, als der letztere. Dass auch Kaninchen der Wirkung der chlorsauren Salze unterliegen, ist von Stokvis selbst bewiesen, aber es gehören dazu grössere Gaben, nach $S$ tokvis 8 bis $12 \mathrm{~g}$ Natr. chlor. pro 1 Kilo, während beim Hund nicht viel mehr als $1 \mathrm{~g}$, beim Menschen, besonders bei Kindern, wie es scheint, noch viel weniger bei einmaliger Einfuhbrung genügt, um den Tod herbeizuführen. Ausserdem kommen bei den am Kaninchen angestellten Versuchen jedenfalls noch ganz andere Umstände, besonders die nachtheilige Einwirkung grosser Flüssigkeitsmengen, in Betracht.

Auf welchem Umstande in letzter Instanz diese, wie ich gern zugebe, sehr auffällige Widerstandsfähigkeit der Kaninchen beruht, entzieht sich vorläufig meiner Beurtheilung. Man könnte vielleicht die Vermuthung aussprechen, dass der grössere Alkalireichthum des Blutes der Pflanzenfresser einen gewissen Einfluss hätte.

Riess hat bereits die Möglichkeit hervorgehoben, dass an der eigenthümlichen Blutzerstörung die Verminderung der Alkalescenz des Blutes die Schuld trage, indem er sich darauf bezieht, dass Friedländer in mehreren Fällen acuter Intoxication durch chlorsaures Kali eine saure Reaction des Leichenblutes fand. Letzteres wirde an sich nicht sehr viel beweisen, da es sich um Leichenblut handelte. Auch würde die Verminderung der Alkalescenz allein die eigenthümliche Blutzerstörung im Sinne von Riess nicht erklären, abgesehen davon, dass es sich bei dieser letzteren bereits um einen Folgezustand handelte, wie ich oben gezeigt habe. Wohl aber könnte eine verminderte Alkalescenz des Blutes den Eintritt der primären Blutveränderung begïnstigen und eine Vermehrung der Alkalescenz denselben verzögern oder gar verhindern.

Dass der Grad der Alkalescenz des Blutes einen Einfluss auf die Einwirkung des Salzes hat, scheint in der That aus den Versuchen v. Mering's mit Blut ausserhalb des Organismus hervorzugehen. v. Mering hat gezeigt, dass Vermehrung der Kohlensäure, sowie Zusatz von saurem phosphorsauren Natron (innerhalb physiologischer Grenzen) die Fähigkeit des Blutes, bei Anwesenheit von chlorsauren Salzen Methämoglobin zu bilden, wesentlich erhöht, während andererseits Zusatz von Alkalien dieselbe vermindert.

Indess wäre es tibereilt, hieraus ohne genauere Untersuchungen bereits bestimmte Schlüsse zu ziehen, wir müssen also vorläufig diese Frage unentschieden lassen. Jedenfalls hängt dieselbe eng zusammen mit der Hauptfrage, welcher Art die Wechselwirkung zwischen chlorsauren Salzen und Blut ist. 
Während ich auf Grund der thatsächlich nachgewiesenen Methämoglobinbildung unter der Einwirkung der chlorsauren Salze annehmen zu müssen glaubte, dass eine Zersetzung der letzteren unter Abgabe von Sauerstoff stattfinden müsse, obwohl eine solche bis dahin nicht nachgewiesen war, und diese Annahme durch den Nachweis der Reduction des Chlorates durch eine reine Lösung von O-Hglb. zu stitzen suchte, beweist Stokvis ron Neuem, dass eine solche Zersetzung im Körper nicht stattfindet, da die Menge der im Harn ausgeschiedenen Chlorate so gut wie vollständig der eingeführten Menge entspreche. Die thatsächliche Richtigkeit seiner Versuche bestreite ich natülich nicht im Mindesten. Doch steht mit denselben

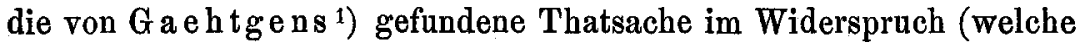
auch Stokvis anfübrt, ohne darauf, wie es scheint, viel Gewicht zu legen), dass im Organismus des Hundes das Kaliumchlorat zu einem beträchtlichen Theile $(1 / 5-1 / 4$ der Einnahme oder absolut ca. $2 \mathrm{~g}$ in 24 Stunden) reducirt wird, sowie die von $v$. Mering ${ }^{2}$ ) mitgetheilte Angabe, dass im Blute eines Hundes, welches nach der Injection von $5 \mathrm{~g}$ Natriumchlorat in die Vena jugularis dunkelbraun geworden war, beträchtlich mehr Chlornatrium enthalten war, als vor der Injection, woraus auf eine Reduction von 0,0131 g chlorsauren Natrons durch $10 \mathrm{ccm}$ Blut geschlossen werden musste.

Diesen allerdings nur vereinzelten Untersuchungsergebnissen stehen zahlreiche andere mit entgegengesetztem Resultate gegenüber, und es wäre daher denkbar, dass es sich in jenen um Beobachtungsfehler gehandelt habe, obwohl dieselben von sorgfältigen und sachverständigen Forschern herrlihren.

Aber es wäre möglicherweise noch eine andere Erklärung der abweichenden Untersuchungsergebnisse zulässig. Es ist zweifellos, dass dieselbe Chloratmenge, welche bei einmaliger Einführung den Tod unter charakteristischen Erscheinungen zur Folge hat, in kleinen mebrfach wiederholten Gaben gar keinen Nachtheil anrichtet. Schnelligkeit der Resorption, wie sie im nüchternen Zustande vom Magen aus, oder bei subcutaner, oder intraperitonealer Einführung stattfindet, begünstigt den Eintritt der toxischen Erscheinungen zweifellos. So-

1) Berliner klin. Wochenschrift. 1883. Nr. 20. Eine ausführlichere Mittheilung des Versuches ist, wie es scheint, noch nicht erfolgt.

2) 1. c. S. 125. Wenn v. Mering bei Gelegenheit dieses Versuches annimmt, dass ein gewisser Methämoglobingehalt des Blutes ohne Schaden ertragen werden könne, so kann ich dem nicht vollständig beistimmen; da der Hund bereits 5 Stunden nach der Injection getödtet wurde, so ist noch nicht sicher, was später daraus geworden wäre. 
mit ist als sicher anzunehmen, dass zur Entfaltung der letzteren eine gewisse Concentration des Salzes im Blute erforderlich ist, eine Annahme, welche auch durch Versuche von v. Mering mit Blutproben ausserhalb des Organismus bestätigt $\mathrm{zu}$ werden scheint.

Wird eine gewisse Concentration des Salzes im Blute nicht erreicht, so wird dasselbe ausgeschieden, ohne dass eine Spur einer toxischen Wirkung eintritt. Es wäre denkbar, wenn auch schwer erklärlich, dass auch die Zersetzung des Salzes im Blute erst bei einer gewissen Concentration ihren Anfang nehme, so dass also die toxischen Erscheinungen einträten, wenn dieser Punkt erreicht wäre. Der Nachweis des Ueberganges der ganzen Chloratmenge in den Harn in unzersetztem Zustande würde dann nur beweisen, dass noch keine Vergiftungserscheinungen im Blute eingetreten waren, dass also die Concentration des Salzes noch nicht den nöthigen Grad erreicht hatte, um die eigenthümliche Wechselwirkung zwischen Hämoglobin und Chlorat herbeizuführen.

Volle Beweiskraft würde der Vergleich der eingefuhrten und der ausgeschiedenen Chloratmenge nur in einem Versuche haben, in welchem nach eingetretener intensiver Blutveränderung die Gesammtmenge der ausgeschiedenen Chlorate bestimmt würde. Und selbst hierbei könnte es sich möglicherweise um sehr geringe Differenzen handeln. Bis jetzt ist der Versuch in dieser Weise noch nicht ausgeftuhrt worden.

Indess hat die ganze Frage von der Zersetzung oder Nichtzersetzung der Chlorate im Blute ein mehr pharmakologiseh-chemisches Interesse und berührt zunächst die pathologische Thatsache der Veränderung des Blutfarbstoffes und der Zerstörung der rothen Blutkörperchen mit ihren Folgen gar nicht; diese letztere lässt sich eben nicht wegleugnen, auch wenn der Nachweis geführt wird, dass eine Zersetzung der chlorsauren Salze im Blute thatsächlich nicht stattfindet. Es würde sich in diesem Falle nur darum handeln, wie die unbestreitbaren pathologischen Veränderungen auf andere Weise zu erklären sind, und man miisste hierbei nicht allein auf die chlorsauren Salze, sondern auch auf die ubrigen Substanzen Rücksicht nehmen, welche die gleiche Eigenschaft haben, im circulirenden Blute Methämoglobin zu bilden.

Um diese Einwirkung ganz zu verstehen, müssten wir zunächst besser über die chemische Natur des Methämoglobin unterrichtet sein, als es zur Zeit der Fall ist.

Jäderholm ${ }^{1}$ ) erklärte bekanntlich das Methämoglobin für Par-

1) Ich erlaube mir hier zu bemerken, dass mir die Arbeit Jäd erholm's zur Zeit meiner Untersuchungen leider gar nicht bekannt war, so dass mir erst 
oxyhämoglobin, indem er annahm, dass dasselbe mehr Sauerstoff enthalte als 0-Hglb. Ich selbst 1) war auf Grund der Umwandlung des reducirten Hämoglobin in Methämoglobin, ohne die Zwischenstufe des 0 -Hglb. (bei Ausschluss von Sauerstoff) zu dem Schluss gekommen, dass das Methämoglobin ein Oxydationsproduct des reducirten Hglb. sei, welches weniger 0 enthalte als das $0-H g l b$. Sa arbach ${ }^{2}$ ) ist dagegen $\mathrm{zu}$ dem Resultat gelangt, dass das Methämoglobin einen höheren 0 -Gehalt als das 0 -Hglb. besitze, und dass dasselbe bei vorsichtiger Reduction durch S-Ammon bei Abschluss von Sauerstoff querst in $\mathrm{O}-\mathrm{Hglb}$. und dann erst in reducirtes $\mathrm{Hglb}$. uibergefuhrt werde. Dagegen haben $H \mathfrak{u} f \mathrm{n}$ er und $\mathrm{R}$. $\mathrm{K} \ddot{u} \mathrm{lz}^{3}$ ) den Nachweis geführt, dass das Methämoglobin nicht mehr und nicht weniger Sauerstoff enthält als das 0-Hglb. Jedenfalls ist aber anzunehmen, dass der Sauerstoff im Methämoglobin auf andere Art gebunden ist als im O-Hglb. und zwar fester.

In welcher Weise nun diese Umwandlung des O-Hglb. unter der Einwirkung von Oxydationsmitteln und anderen Substanzen, welche wohl kaum als solche zu betrachten sind, zu Stande kommt, das ist eine Frage, deren Entscheidung vielleicht noch lange ihrer Lösung harren wird. Jedenfalls ist man aber nicht berechtigt, mit Stokvis zu schliessen: Da keine Zersetzung der chlorsauren Salze im lebenden Körper nachgewiesen werden kann, kann auch kein Methämoglobin sich bilden, also kann dieses, wo es vorkommt, nur Folge einer cadaverösen Veränderung sein. Der Versuch und die Vergiftungsfälle beim Menschen setzen die Bildung des Methämoglobin im lebenden Blute ausser Zweifel; unmittelbare Folge derselben ist entweder der Tod oder die Ausscheidung der zerstörten Blutkörperchen und des gelösten Farbstoffes durch die Nieren, resp. Anhäufung in der Milz und weitere Veränderungen.

Wenn ich auch glaube, dass ich durch die vorhergehende, vielleicht schon zu ausfubrliche Auseinandersetzung hinreichend nachgewiesen habe, dass der Angriff Stokvis' gegen die Richtigkeit der von mir behaupteten Thatsachen ungerechtfertigt war, und dass dieselben für den Menschen, um den es sich doch in erster Linie handelt, und für den Hund ihre volle Gültigkeit behalten, so mochte ich

nach vielfachen Bemühungen die Feststellung gelang, dass der im Blute sich bildende Körper das damals noch wenig bekannte Methämoglobin sei und nicht saures Hämatin, wie ich anfangs vermuthet hatte.

1) Ueber das Methămoglobin. Virchow's Archiv. 77. Bd. S. 493.

2) Archiv für die gesammte Physiologie. XXVIII. Bd. S. 382.

3) Zeitschrift für physiologische Chemie. 
232 X. MARCHAND, Ueber die giftige Wirkung der chlorsauren Salze.

mich doch nicht hierbei begnügen, um so weniger, als der Angriff von so angesehener Seite kam, dass ich fürchten musste, dass es dem Urheber nicht an vielseitiger Anerkennung fehlen und dadurch andererseits auch ein verderblicher Einfluss auf den ohnehin schon sehr lax gehandhabten Verbrauch des chlorsauren Kali als Volksheilmittel ansgetibt werde. Die Folge von übermässig warmen Empfehlungen und der Geringschätzung der giftigen Wirkung eines Mittels pflegt, wie wir dies gerade bei dem chlorsauren Kali beobachten konnten, eine grössere Anzahl von Vergiftungsfällen zu sein, deren Verantwortung zum grossen Theil auf die Urheber jener Meinung zuriuckfällt. Abgesehen davon lag es mir daran, einige noch mangelhaft aufgeklärte oder streitige Punkte in Betreff der pathologischen Veränderungen unter dem Einfluss der chlorsauren Salze durch einige neue Versuche womöglich aufzuklären.

(Fortsetzung folgt.) 